jz-2021-03842u.R1

Name: Peer Review Information for "Glassy or Amorphous? A Demonstration from G-phase Copper Containing Fivefold Twining Structure"

First Round of Reviewer Comments

Reviewer: 1

Comments to the Author

The ms by Liu et al is a significant contribution to a most interesting scientific debate about a newly found intermittant phase (called G-phase or glacial phase) in between the liquid and the crystalline phase.

The authors worked on monoatomic Cu systems using MD simulations and studied the structure, the thermodynamics and time dependent evolution of this new $\mathrm{G}$-phase. They conclude it is not a fully liquid or frozen in liquid state, which is commonly called a glass, nor a crystal but what they call a mesophase. They compare their work with published work by An et al. and agree with this literature in the findings but analyse carefully the structure and find a new FFTS mesoscopic order. They disagree in the wording calling this a glass because their is not classical glass transition and the $\mathrm{G}$ phase melts directly back into the liquid indicating also no crystallisation step .

So far so good. The ms is a careful study on this new phase found also in experiments in the meantime. The ms is for me to much about wording. Not calling the G-phase a glass ( implementing this is a frozen liquid) is fine. But also amorphous thin films never showed a glasstransition ( if monoatomic) but crystallised even at cryogenic temperatures into a crystal and everybody agreed to call it amorphous. Secondly the composition dependence at the end of the ms is off. No composition dependence is studied here. The authors should take it out and do their homework first on this.

Nevertheless the ms should be publised after these small corrections and it will contribute significantly to this new and lively debate.

Reviewer: 2

\title{
Comments to the Author
}

The authors present a molecular dynamics study on $\mathrm{Cu}$ to investigate the structure and thermodynamics of different phases/microstructures formed upon rapid quenching. The work is motivated by a recent publication that proposed the existence of a so-called G-phase, which is a different structural realization of an amorphous structure than the true glass, here referred to an L-glass. The paper is well written, has a clear goal in raising an important question related to the most recent developments in the field, and is needed to advance the scientific discussion on possible other amorphous and metastable phases than so far considered in the field of metallic glasses. 
The paper essentially raises the question what a glass really is. Here, the authors make a good discrimination between systems than undergo a true glass transition and those that have a disordered structure (lack of long-range order) but exhibit no true glass transition.

I find the work very stimulating, and one aspect caught in particular my attention. The authors report that the $\mathrm{G}$ phase is a metastable phase occurring in the middle of the real glass $(\mathrm{L})$ to crystal $(\mathrm{X})$ transition. To me, this indicates that we are looking at a particular structural realization resulting from an incomplete first order transition. As such, I would actually not be surprised if it later comes out that the here called $G$ phase is indeed some intermediate and possibly non-equilibrium structural part of the trajectory from $L$ to $X$. In that context, it is worthwhile to consult this recent work: Journal of Alloys and Compounds 821 (2020) 153209, where indeed several of the authors discussed points are also addressed.

Looking at the data for the $G$ phase, I am much reminded of nanocrystalline metals. When the structural analysis is done, have the authors considered allowing a certain degree of strain away from ideal fcc or hcp regions? This structure could also be a rather heavily strained nanocrystalline material.

Along these lines, it is of course interesting that the $\mathrm{G}$ phase seems to be relatively stable. Here I am reminded of amorphous grain boundary complexions known to form in CuZr systems. The same interesting situation emerges, where a rather stable several nanometer thick amorphous interface forms in between crystalline grains (Acta Materialia 206 (2021) 116650). Whilst this can be understood to some degree in multicomponent systems, where particular species are left behind and thereby allow the formation of an amorphous interface (or even matrix) surrounding crystallites, it remains unclear to me how this can be a stable structural state for a monoatomic system. See again the above quoted paper in JALCOM.

I am somewhat puzzled by the schematic of figure 5 . I find it a bit speculative to set various phases at the same free energy and it is unclear to me how the authors have compelling evidence to add a composition dimension to the discussed problem.

Overall, I think this paper is an excellent contribution to the field.

Author's Response to Peer Review Comments:

Manuscript ID: jz-2021-03842u

Title: "Glassy or Amorphous? A Demonstration from G-phase Copper Containing Five-fold Twining Structure"

\section{Dear EDITOR,}

We sincerely thank you for considering our manuscript and allowing us to revise. We appreciate the comments and suggestions made by the reviewers and are pleased to hear that all the reviewers liked the paper and considered further review is not needed. Minor corrections have been made including the removal of Fig. 5 and adding an explanation to discussion in answering reviewer \#2's question. Point-by-point replies to each of the reviewers' question are given below, and a list of the corresponding revised places of the manuscript, highlighted in the 
revision, is attached to the end of the response letter. We hope that the revised manuscript is suitable to be published in the Journal of Physical Chemistry Letters.

Sincerely Yours,

$$
\text { Yonghao Sun }
$$

Professor Y. H. Sun (on behalf of the authors).

$2022 / 1 / 5$

\section{Responses to Reviewer \#1}

Reviewer \#1: The ms by Liu et al is a significant contribution to a most interesting scientific debate about a newly found intermittant phase (called G-phase or glacial phase) in between the liquid and the crystalline phase. The authors worked on monoatomic $\mathrm{Cu}$ systems using MD simulations and studied the structure, the thermodynamics and time dependent evolution of this new $\mathrm{G}$-phase. They conclude it is not a fully liquid or frozen in liquid state, which is commonly called a glass, nor a crystal but what they call a mesophase. They compare their work with published work by An et al. and agree with this literature in the findings but analyse carefully the structure and find a new FFTS mesoscopic order. They disagree in the wording calling this a glass because their is not classical glass transition and the $G$ phase melts directly back into the liquid indicating also no crystallisation step .

Response: We thank the reviewer for summarizing the key points of our work.

Reviewer \#1: So far so good. The ms is a careful study on this new phase found also in experiments in the meantime. The ms is for me to much about wording. Not calling the G-phase a glass (implementing this is a frozen liquid) is fine. But also amorphous thin films never showed a glass transition (if monoatomic) but crystallised even at cryogenic temperatures into a crystal and everybody agreed to call it amorphous.

Response: We have no dispute with the reviewer in terms of terminology and agreed that both "amorphous" and "glassy" materials are able to crystallize. However, we highlighted the difference between "amorphous" and "glassy" because we found many possibilities for the mesophases between crystal and glass exist than just G-glass. Distinguishing "amorphous" and "glassy" is thus useful in understanding the G-phase.

Reviewer \#1: Secondly the composition dependence at the end of the ms is off. No composition dependence is studied here. The authors should take it out and do their homework first on this. Response: We agree with the reviewer and have taken Fig. 5 out of the manuscript. 
Reviewer \#1: Nevertheless the ms should be publised after these small corrections and it will contribute significantly to this new and lively debate.

Response: Thank you again for your recommendation.

\section{Responses to Reviewer \#2}

Reviewer \#2: The authors present a molecular dynamics study on Cu to investigate the structure and thermodynamics of different phases/microstructures formed upon rapid quenching. The work is motivated by a recent publication that proposed the existence of a socalled G-phase, which is a different structural realization of an amorphous structure than the true glass, here referred to an L-glass. The paper is well written, has a clear goal in raising an important question related to the most recent developments in the field, and is needed to advance the scientific discussion on possible other amorphous and metastable phases than so far considered in the field of metallic glasses.

Response: We thank the reviewer for emphasizing the importance of our work.

Reviewer \#2: The paper essentially raises the question what a glass really is. Here, the authors make a good discrimination between systems than undergo a true glass transition and those that have a disordered structure (lack of long-range order) but exhibit no true glass transition. I find the work very stimulating, and one aspect caught in particular my attention. The authors report that the $\mathrm{G}$ phase is a metastable phase occurring in the middle of the real glass $(\mathrm{L})$ to crystal $(X)$ transition. To me, this indicates that we are looking at a particular structural realization resulting from an incomplete first order transition. As such, I would actually not be surprised if it later comes out that the here called G phase is indeed some intermediate and possibly non-equilibrium structural part of the trajectory from $L$ to $X$. In that context, it is worthwhile to consult this recent work: Journal of Alloys and Compounds 821 (2020) 153209, where indeed several of the authors discussed points are also addressed.

Response: We thank the reviewer for pointing out this newly-released reference paper. It is included in the discussion.

Reviewer \#2: Looking at the data for the G phase, I am much reminded of nanocrystalline metals. When the structural analysis is done, have the authors considered allowing a certain degree of strain away from ideal fcc or hcp regions? This structure could also be a rather heavily strained nanocrystalline material. Along these lines, it is of course interesting that the $G$ phase seems to be relatively stable. Here I am reminded of amorphous grain boundary complexions known to form in CuZr systems. The same interesting situation emerges, where a rather stable several nanometer thick amorphous interface forms in between crystalline grains (Acta Materialia 206 (2021) 116650). Whilst this can be understood to some degree in multicomponent systems, where particular species are left behind and thereby allow the 
formation of an amorphous interface (or even matrix) surrounding crystallites, it remains unclear to me how this can be a stable structural state for a monoatomic system. See again the above quoted paper in JALCOM.

Response: We do not consider the G-phase $\mathrm{Cu}$ as heavily strained nanocrystalline materials. We have considered the strain effects: the fcc or hcp regions are recognized by the PTM algorithm (not the CNA method), which allows a root-mean-squared-deviation as large as 0.12 (the default value is 0.1 in many previous studies. See \$Methods). Also, the hcp planes of the Fivefold twining structures are not always flat: some have curvatures, indicating strong elastic distortion. However, we think the stability of the FFTS comes from the growth inability of the clusters under the 5-fold symmetry, which is different from multi-component systems allowing chemical segregation. Such 5-fold symmetry is forbidden in crystalline structures opposing the possibility of nano-crystals. This point is added to discussion.

Reviewer \#2: I am somewhat puzzled by the schematic of figure 5 . I find it a bit speculative to set various phases at the same free energy and it is unclear to me how the authors have compelling evidence to add a composition dimension to the discussed problem. Overall, I think this paper is an excellent contribution to the field.

Response: We agree with the reviewer and have removed Fig. 5 from the manuscript.

\section{List of changes to the manuscript}

(Highlighted with a yellow background in the revised manuscript)

1. Fig. 5 is removed.

2. We added an explanation on why $\mathrm{G}$-phase $\mathrm{Cu}$ is not a heavily deformed crystal to Discussion in order to address Reviewer 2's comments. 